Deniz Öğütmen Koç*, Yağmur Özhan, Ebru Türköz Acar, Nilgün Bireroğlu, Fatih Aslan, Murat Keğin, Hande Sipahi

\title{
Serum Neopterin Levels and IDO Activity as Possible Markers for Presence and Progression of Hepatitis B
}

https://doi.org/10.1515/pteridines-2020-0010

received July 20, 2018; accepted August 22, 2018.

\begin{abstract}
Chronic Hepatitis B virus (HBV) is still one of the major reasons for liver related mortality and morbidity all around the world. This study aimed to investigate the possible relationship between the immune system activation and presence, as well as progression, of hepatitis $\mathrm{B}$ infection by monitoring the tryptophan degradation and serum neopterin levels in patients with HBV. 110 patients with HBV and 23 healthy subjects were included in the study. The patients had significantly higher neopterin levels and increased kynurenine to tryptophan ratios, which were most probably due to enhanced indoleamine 2,3-dioxygenase (IDO) activity compared to healthy control. A strong positive correlation was found between neopterin levels and IDO activity in patient group. Neopterin levels and IDO activity were markedly increased in patients with histological activity index (HAI) $\geq 4$ compared to $\mathrm{HAI}<4$, and a significant correlation was found between neopterin and HAI. Moreover, there
\end{abstract}

*Corresponding author: Deniz Öğütmen Koç, Department of Gastroenterology, University of Health Sciences, Gaziosmanpaşa Training and Research Hospital, 34255, Gaziosmanpaşa, Istanbul, Turkey, E-mail: drdenizkoc@gmail.com

Yağmur Özhan, Hande Sipahi, Department of Pharmaceutical Toxicology, Faculty of Pharmacy, Yeditepe University, 34755, Atasehir, Istanbul, Turkey

Ebru Türköz Acar, Department of Analytical Chemistry, Faculty of Pharmacy, Yeditepe University, 34755, Atasehir, Istanbul, Turkey Nilgün Bireroğlu, Department of Biochemistry, University of Health Sciences, Gaziosmanpaşa Training and Research Hospital, 34255, Gaziosmanpaşa, Istanbul, Turkey

Fatih Aslan, Department of Internal Medicine, University of Health Sciences, Gaziosmanpaşa Training and Research Hospital, 34255, Gaziosmanpaşa, Istanbul, Turkey

Murat Keğin, Department of Surgery, University of Health

Sciences, Gaziosmanpaşa Training and Research Hospital, 34255,

Gaziosmanpaşa, Istanbul, Turkey was a significant correlation between albumin levels and IDO activity in HBV patients. These findings suggest that tryptophan degradation results from IFN-y-induced IDO activation, likewise depletion of albumin synthesis in HBV patients may result from diminished tryptophan availability. In conclusion, based on the study results, serum neopterin levels and IDO activity could provide additional immunological information for monitoring liver histological activity and can be used as prognostic markers in HBV disease.

Keywords: Hepatitis B virus; tryptophan; kynurenine; neopterin; immune activation.

\section{Introduction}

Hepatitis B virus (HBV) is still an important worldwide health concern. According to World Health Organization's (WHO) data, it is estimated that 257 million people are living with chronic HBV infection and nearly 1 million people are dying because of chronic HBV infection related cirrhosis and hepatocellular carcinoma [1].

Chronic HBV infection, which is characterized by the presence of hepatitis B surface antigen (HBsAg) for at least 6 months in blood plasma, is a dynamic process that reflects the interaction between $\mathrm{HBV}$ replication and host immune response. However, not all chronic HBV infection patients have chronic hepatitis $[2,3]$. According to the latest international guides, chronic HBV infection is classified under following 5 stages: (I) hepatitis Be-antigen (HbeAg) positive chronic infection (HbsAg high, HBV DNA $>10^{7} \mathrm{IU} / \mathrm{mL}$, alanine aminotransferase (ALT) normal, and liver disease none/minimal); (II) HBeAg positive chronic hepatitis (HbsAg high/intermediate, HBV DNA 10 $10^{4}-10^{7}$ $\mathrm{IU} / \mathrm{mL}$, ALT elevated, and liver disease moderate/severe); (III) HBeAg negative chronic infection (HbsAg low, 
HBV DNA <2000 IU/mL, ALT normal, and liver disease none); (IV) HBeAg negative chronic hepatitis (HbsAg intermediate, HBV DNA >2000 IU/mL, ALT elevated, and liver disease moderate/severe); and (V) HBsAg negative phase. Even after a full evaluation, some patients remain in an ambiguous grey area that requires individualized management [2]. Classically, HBV DNA levels are used as a virus replication indicator, and serological indicators and ALT levels are used for the monitoring of chronic HBV infection. Recently, quantitative HBsAg (qHBsAg) levels and HBV DNA levels were found to be correlated. Higher qHBsAg levels are correlated with the progression of cirrhosis and hepatocellular carcinoma [4].

Since hepatitis is characterized by the presence of inflammatory cells in liver tissue, the alteration of inflammation markers is being investigated, and neopterin levels reflecting cellular immune response seem to be increase in hepatitis B infection [5]. Neopterin is mainly synthesized by activated macrophages and reflects the endogenous release of interferon gamma (IFN- $y$ ) $[6,7]$. Neopterin is known to increase in cases of viral, bacterial, and parasitic infections and is a useful marker in early diagnosis of immune alterations as well as the degree and progression of certain diseases $[8,9]$.

Indoleamine-2,3-dioxygenase (IDO), which is also up-regulated by various cytokines in particular IFN-y, have been shown to increase in hepatitis [10]. Tryptophan (Trp) degradation by the immunosuppressive enzyme IDO can be altered by immune modulation and inflammation. Trp is an essential amino acid which is a constituent of proteins and the precursor of two important biosynthetic pathways: the generation of the serotonin neurotransmitter and the formation of kynurenine (Kyn) derivatives [11]. Oxidation of Trp to Kyn is catalyzed by tryptophan pyrrolase (tryptophan 2,3-dioxygenase, TDO) mainly in the liver. However, since TDO activity is inhibited in liver diseases, any increase in the conversion of Trp into Kyn in serum in liver diseases suggested to result from the increase in IDO activity. Hence the plasma Kyn/Trp ratio is frequently used to express the activity of IDO [10].

It is not yet known whether neopterin levels and IDO activity can be considered as biomarkers for the activation stages of the chronic HBV infection. Therefore, in the present study, we aimed to determine a possible relationship between the immune system activation and presence and progression of hepatitis B by monitoring the Trp degradation and serum neopterin levels in patients with chronic hepatitis.

\section{Materials and methods}

\section{Study Groups and Sample Collections}

This study originated with 116 patients ( 65 males, 51 females) infected with the HBV who consecutively referred to the out-patient clinic at the Department of Gastroenterology of the Gaziosmanpaşa Training and Research Hospital. Six patients were excluded from enrollment. Of these patients, 3 patients had hepatitis D infections, 2 had autoimmune hepatitis, and 1 had autoimmune pancreatitis besides the hepatitis B infection. Therefore, 110 patients (60 males, 50 females) were included in the study. We also evaluated 23 healthy (11 males, 12 females) subjects as a control group. The average age of the patients was $43 \pm 9$ years, whereas control group's average age was $40 \pm 7$ years. There was no significant difference between the ages of the groups.

Chronic hepatitis B infection was determined with hepatitis B seroposivity for more than 6 months. Both patient and control groups were without hepatitis C, hepatitis D, human immunodeficiency virus (HIV) infections, systemic bacterial or fungal infection, and autoimmune diseases. Serum HbsAg, ALT, aspartate aminotransferase (AST), gamma-glutamyl transpeptidase (GGT), alkaline phosphatase (AP), and albumin levels along with prothrombin time (PTZ) were determined in all participants. HBV DNA, HbeAg, and Hepatitis B e-antibodies (AntiHBe) levels were analyzed only in the patient group in the hospital biochemistry laboratory.

Patients with HBV infection were divided into subgroups according to their qHBsAg $(<1000$ and $>1000$ $\mathrm{IU} / \mathrm{mL}$ ) levels, HbeAg (positive and negative), and HBV DNA ( $<2000$ and $>2000 \mathrm{IU} / \mathrm{mL}$ ) levels. Hepatitis B related liver cirrhosis was diagnosed by clinical, serological, and biochemical tests or by histopathological examination of liver biopsy. Patients were also divided into two groups according to their histological activity index (HAI $<4$ and 24) to investigate the changes of biomarkers. Liver biopsy samples were evaluated with the widely used scoring system named the Knodell's histologic activity index. Knodell's scoring system was graded between $0-18$, and categorize disease process as 1-3: no activity, 4-8: mild, 9-12: moderate activity, and 13-18: severe activity [12].

Serum samples taken from the subjects were stored at $-20^{\circ} \mathrm{C}$ in the dark until neopterin, Kyn, and Trp level measurement.

Ethical approval: The research related to human use has been complied with all the relevant national regulations, institutional policies and in accordance the tenets of the Helsinki Declaration, and has been approved 
by the authors' institutional review board or equivalent committee.

Informed consent: Informed consent has been obtained from all individuals included in this study

\section{Determination of tryptophan degradation and kynurenine levels}

Serum Trp and Kyn levels were measured by highperformance liquid chromatography (HPLC) [13]. After preparing a stock solution of Kyn and Trp, standard solutions were prepared by dilution of the stock solutions with albumin solution $(7 \mathrm{~g} / \mathrm{mL})$. Then perchloric acid was added to precipitate proteins and the sample was centrifuged at 13,000 $\mathrm{rpm}$ for $6 \mathrm{~min}$. The supernatant was used for analysis. Sample solutions were treated in the same way for HPLC analysis. The amount of Kyn and Trp in the samples were calculated by using the standard solution curve between 0.75 to $10 \mu \mathrm{M}$ and 7.5 to $100 \mu \mathrm{M}$, respectively. The IDO activity of the samples were estimated by the calculated Kyn/Trp ratio expressed as $\mu$ mol Kyn per mmol Trp.

HPLC specifications: The Agilent 1260 Infinity HPLC system (Darmstadt, Germany) was used for the studies. The system included a G1311B quaternary pump, a G1329B standard autosampler, a G1316A thermostatted column compartment, a G4212B diode array detector, and G721B fluorescence detector. The chromatographic data were obtained using Agilent ChemStation software (Rev. B. 04.03-SP2 (105)). The chromatographic separation was carried out on the ACE C18 $(4.6 \times 250 \mathrm{~mm}, 5 \mu \mathrm{m})$ column (United Kingdom). A Phenomenex C18 guard column (Part No: KJ0-4282) was also used to protect the column and system. The column temperature was maintained at $25^{\circ} \mathrm{C}$. Kyn levels was detected by using a DAD detector at $360 \mathrm{~nm}$. Monitoring of the Trp levels were carried on by using a fluorescence detector and for this purpose, $285 \mathrm{~nm}$ wavelength was used for excitation and $365 \mathrm{~nm}$ wavelength was used as for emission. The mobile phase was the mixture of $15 \mathrm{mM} \mathrm{KH}_{2} \mathrm{PO}_{4}$ solution and acetonitrile (ACN) (93:7). The flow rate of the mobile phase was $0.8 \mathrm{~mL}$ $\mathrm{min}^{-1}$. The injection volume was $25 \mu \mathrm{L}$.

\section{Measurement of neopterin levels}

Neopterin concentrations in serum samples were measured following the basic principle of competitive enzyme-linked immunosorbent assay (ELISA) system
(DRG Diagnostics, Germany) according to manufacturer's instructions. Results were expressed as nmol/L.

\section{Statistical analysis}

Results were expressed as mean \pm standard deviation (mean $\pm \mathrm{SD}$ ). The differences among patient and control groups were evaluated with Kruskal-Wallis analysis of variance. The comparison between two independent groups was done using the Mann-Whitney $U$-test. Correlations of the parameters were evaluated using Spearman's nonparametric correlation test. $P$ values of less than 0.05 were considered significant.

\section{Results}

First, all participants were classified into two groups as patients with hepatitis B disease and healthy subjects as a control. As seen in Table 1, AST and ALT levels was nearly two times higher in HBV patients compared to healthy volunteers. When Kyn and Kyn/Trp levels of patients and control group were compared, both markers were found to be significantly higher in the patients with HBV infection $(p<0.001)$. However, the Trp levels of patients were slightly higher than control but not significant. The serum neopterin levels of the patients were significantly higher than control group $(\mathrm{p}<0.001)$.

Then, patients with HBV infection were divided into subgroups according to their $\mathrm{HbsAg}, \mathrm{HbeAg}$, and HBV DNA levels. The mean levels of measured parameters for each subgroup were established and evaluated (Table 1). When the measured parameters were evaluated based on HBV DNA $<2000 \mathrm{IU} / \mathrm{mL}$ and $>2000 \mathrm{IU} / \mathrm{mL}$, both AST and ALT levels were significantly higher in patients with HBV DNA $>2000 \mathrm{IU} / \mathrm{mL}$ than those with HBV DNA $<2000 \mathrm{IU} /$ $\mathrm{mL}(\mathrm{p}<0.001)$. There were no differences in neopterin and Kyn/Trp levels between the two groups. When patients were grouped according to their HbsAg levels $(<1000 \mathrm{IU} /$ $\mathrm{mL}$ and $>1000 \mathrm{IU} / \mathrm{mL}$ ), there was no difference between the groups in terms of the levels of ALT, AST, Kyn/Trp, and neopterin. Finally, the patients with HbeAg negative and positive were evaluated. The patients with $\mathrm{HbeAg}$ positive were found to have significant higher AST and ALT levels compared to the patients with HbeAg negative ( $p<0.001)$, but the other measured parameters were not different between the subgroups.

The relationship between HAI index of the patients (HAI $<4$ and $\geq 4$ ) and the change of biomarkers was also 
Table 1: Distribution of numbers of the participants by subgroups and concentrations of the measured parameters.

\begin{tabular}{|c|c|c|c|c|c|c|c|}
\hline \multirow[t]{2}{*}{ Groups } & \multirow[t]{2}{*}{$n=133$} & $\begin{array}{l}\text { AST } \\
\text { (IU/L) } \\
\end{array}$ & $\begin{array}{l}\text { ALT } \\
\text { (IU/L) }\end{array}$ & $\begin{array}{l}\text { Kynurenine } \\
(\mu \mathrm{mol} / \mathrm{L})\end{array}$ & $\begin{array}{l}\text { Tryptophan } \\
(\mu \mathrm{mol} / \mathrm{L})\end{array}$ & $\begin{array}{l}\text { Kyn/Trp ( } \mu \mathrm{mol} / \\
\mathrm{mmol})\end{array}$ & $\begin{array}{l}\text { Neopterin } \\
(\mathrm{nmol} / \mathrm{L})\end{array}$ \\
\hline & & \multicolumn{6}{|c|}{ Mean \pm SD $(\min -\max )$} \\
\hline Control & $n=23$ & $15.52 \pm 3.86$ & $13.83 \pm 5.22$ & $4.15 \pm 1.22$ & $91.5 \pm 25.84$ & $45.80 \pm 9.05$ & $7.19 \pm 1.76$ \\
\hline Patients & $n=110$ & $37.57 \pm 54.62^{\star \star}$ & $51.26 \pm 108.47^{\star \star}$ & $5.65 \pm 1.63^{\star \star}$ & $99.95 \pm 20.61$ & $57.35 \pm 12.86^{\star *}$ & $10.88 \pm 3.38^{\star \star}$ \\
\hline \multicolumn{8}{|l|}{ HBV-DNA } \\
\hline$<2000$ & $\mathrm{n}=64$ & $27.75 \pm 14.65^{\star \star}$ & $32.22 \pm 27.39^{\star *}$ & $5.87 \pm 1.82^{\star \star}$ & $102.67 \pm 20.63$ & $58.13 \pm 13.45^{\star \star}$ & $11.36 \pm 3.34^{\star *}$ \\
\hline$>2000$ & $n=46$ & $52.48 \pm 82.84^{\star \star A}$ & $80.34 \pm 165.1^{\star \star B}$ & $5.33 \pm 1.28^{\star \star}$ & $95.93 \pm 20.15$ & $56.33 \pm 12.11^{\star *}$ & $10.25 \pm 3.38^{\star *}$ \\
\hline \multicolumn{8}{|l|}{ HbsAg } \\
\hline$<1000$ & $n=15$ & $25.92 \pm 7.61^{\star \star}$ & $26.77 \pm 14.44^{\star}$ & $5.30 \pm 1.44^{\star}$ & $96.40 \pm 20.32$ & $54.96 \pm 11.17^{\star}$ & $10.96 \pm 3.69^{* *}$ \\
\hline$>1000$ & $n=95$ & $39.13 \pm 57.96^{\text {** }}$ & $54.55 \pm 115.07^{\star *}$ & $5.69 \pm 1.66^{\star *}$ & $100.43 \pm 20.71$ & $57.70 \pm 13.11^{* *}$ & $10.88 \pm 3.38^{\star \star}$ \\
\hline \multicolumn{8}{|l|}{ HbeAg } \\
\hline Negative & $\mathrm{n}=97$ & $37.47 \pm 58.39^{\star *}$ & $50.03 \pm 115.83^{\star * *}$ & $5.62 \pm 1.69^{\star \star}$ & $100.37 \pm 21.30$ & $56.86 \pm 13.05^{\star *}$ & $10.90 \pm 3.12^{\star *}$ \\
\hline Positive & $n=13$ & $36.69 \pm 7.99^{\star \star A}$ & $60.00 \pm 23.62^{\star \star c}$ & $5.86 \pm 1.21^{\star *}$ & $96.84 \pm 14.83$ & $61.02 \pm 11.77^{\star \star}$ & $10.97 \pm 4.76^{\star *}$ \\
\hline
\end{tabular}

${ }^{*} p<0.05,{ }^{\star *} p<0.001$, compared to control; ${ }^{A} p<0.05,{ }^{B} p<0.01,{ }^{C} p<0.001$, comparing the difference between subgroups. AST: aspartate aminotransferase, ALT: alanine aminotransferase, Kyn/Trp: kynurenine/tryptophan ratio, HBV DNA: Hepatitis B virus DNA (IU/mL), HBsAg: hepatitis B surface antigen (IU/mL), HBeAg: hepatitis B e-antigen.

examined (Figure 1). Compared to patients with HAI <4, serum neopterin concentration was markedly increased in patients with $\mathrm{HAI} \geq 4(8.42 \pm 0.60$ vs. $11.15 \pm 4.31 \mathrm{nmol} / \mathrm{L}$, p<0.01) (median $8.45 \mathrm{nmol} / \mathrm{L}$, min-max: $7.65-9.24 \mathrm{nmol} / \mathrm{L}$ vs. median $10.01 \mathrm{nmol} / \mathrm{L}$, min-max: 7.06-27.23 nmol/L). IDO activity was also significantly higher in patients with $\mathrm{HAI} \geq 4(60.57 \pm 11.24 \mu \mathrm{mol} / \mathrm{mmol})$, compared to patients with HAI <4 (51.15 $\pm 9.42 \mu \mathrm{mol} / \mathrm{mmol}, \mathrm{p}<0.05)$ (median $52.20 \mu \mathrm{mol} / \mathrm{mmol}$, min-max: $37.23-66.33 \mu \mathrm{mol} / \mathrm{mmol}$ vs. median $56.69 \mu \mathrm{mol} / \mathrm{mmol}$, min-max: 43.37-87.87 $\mu \mathrm{mol} /$ mmol).

The correlation between biochemical parameters in patients was evaluated and the results are shown in Table 2. Considering all the values, there was a significant correlation between serum neopterin and Kyn/Trp concentration in the patient group. There was also a significant positive correlation between neopterin levels and HAI levels of HBV patients. Kyn/Trp and albumin level were significantly negatively correlated.

The correlation between Trp, Kyn, Kyn/Trp, and neopterin levels were also evaluated for the patients divided into subgroups according to their $\mathrm{HbsAg}$, HbeAg, and HBV DNA levels (Table 3). We could only find significant positive correlation between neopterin and HbeAg levels for the patients HBeAg negative. There was a significant negative correlation between Kyn/Trp ratio and the patients with HBV DNA lower than 2000.

\section{Discussion}

Chronic hepatitis B is still one of the major reasons for liver related mortality and morbidity all around the world. If not treated, one third of patients develop serious diseases such as liver failure, cirrhosis, or hepatocellular carcinoma at their last stages [14]. The presence of HBsAg constitutes the diagnosis of hepatitis B and the presence of the antibody in the blood stream for more than 6 months refers to chronic hepatitis. Moreover, high levels of HBV DNA in the plasma have been closely related to the development of liver failure, cirrhosis, or hepatocellular carcinoma [15]. Turkey is in the medium endemicity zone in terms of $\mathrm{HBV}$ infection and since the frequency of $\mathrm{HBsAg}$ is between 0.8 to $5.7 \%$, the treatment and monitoring of chronic HBV patients is crucial [16].

As it is known that HBV is a non-lytic virus that does not cause cell damage directly. In HBV infection, the host cellular immune response to viral antigens is reported to cause liver damage and viral clearance [17]. Immune system mediated hepatic necrosis is a result of the attack of host cytotoxic T cells to HBV infected hepatocytes [3]. In HBV infection, stimulation of $\mathrm{T}$ lymphocytes by viral antigens increases the production of IFN- $y$ that activates macrophages in the liver and stimulates neopterin release from these macrophages $[18,19]$. Similarly, in our result significantly higher levels of neopterin in the patients 

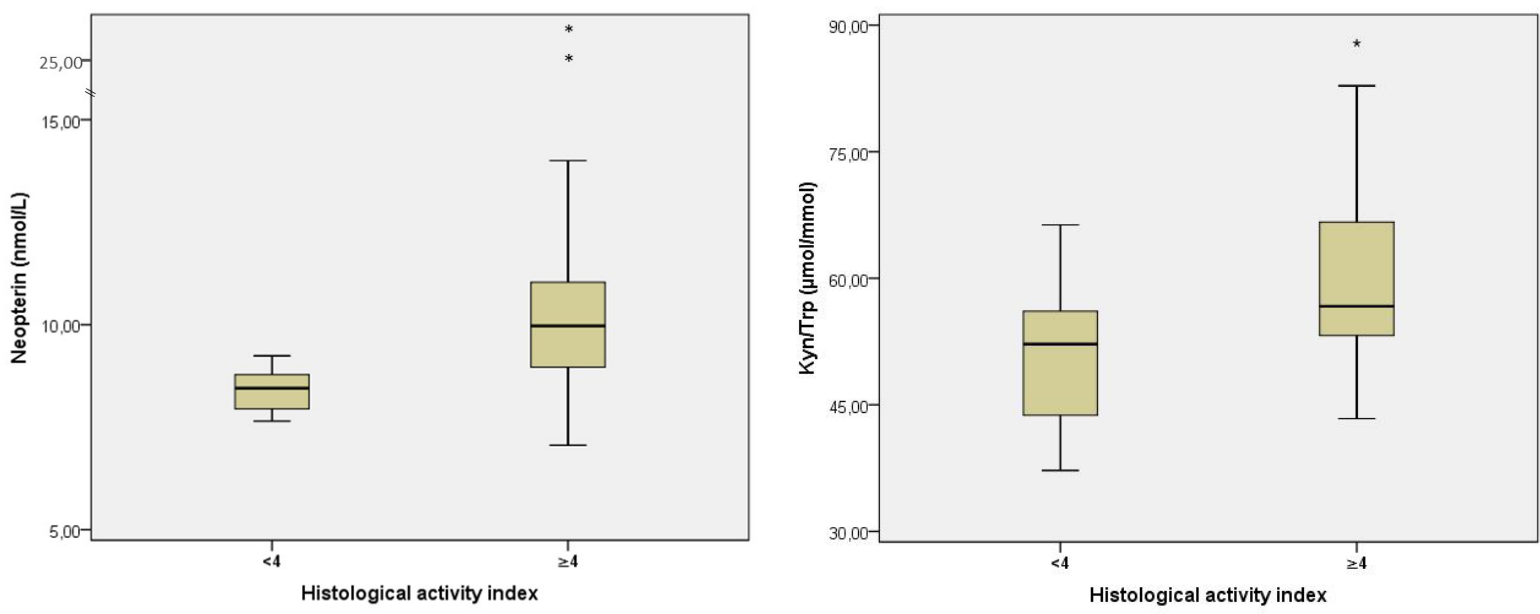

Figure 1: The comparison of serum concentration of neopterin and IDO activity in patients with $\mathrm{HAI}<4$ and $\mathrm{HAI} \geq 4$.

HAI: histological activity index. The upper and lower portions of the boxes indicate $25^{\text {th }}$ and $75^{\text {th }}$ percentiles, respectively. Horizontal lines within the boxes indicate the $50^{\text {th }}$ percentile (median).

Table 2: Correlations between the parameters in HBV patients.

\begin{tabular}{|c|c|c|c|c|c|}
\hline \multirow[t]{2}{*}{ HBV Patients } & \multicolumn{5}{|c|}{ Spearman correlation coefficients (p-value) } \\
\hline & Trp & Kyn & Kyn/Trp & HAI & Albumin \\
\hline Neopterin & $-0.039(0.751)$ & $-0.055(0.653)$ & $0.304(0.011)^{\star}$ & $0.391(0.018)^{\star}$ & $-0.055(0.690)$ \\
\hline $\operatorname{Trp}$ & & $0.719(<0.001)^{\star}$ & $-0.232(0.015)^{\star}$ & $0.135(0.366)$ & $0.166(0.142)$ \\
\hline Kyn & & & $0.758(<0.001)^{\star}$ & $0.281(0.059)$ & $-0.253(0.023)^{\star}$ \\
\hline Kyn/Trp & & & & $0.156(0.301)$ & $-0.394(<0.001)^{\star}$ \\
\hline HAl & & & & & $-0.153(0.366)$ \\
\hline
\end{tabular}

*. Correlation is significant at the 0.05 level;

Kyn/Trp: kynurenine/tryptophan ratio; HAl: histological activity index.

Table 3: Correlations for Trp, Kyn, Kyn/Trp, and neopterin levels in HBV patient subgroups.

\begin{tabular}{|c|c|c|c|c|}
\hline \multirow[t]{2}{*}{ Subgroups } & \multicolumn{4}{|c|}{ Spearman correlation coefficients ( $p$-value) } \\
\hline & Trp & Kyn & Kyn/Trp & Neopterin \\
\hline HBV DNA & $-0,051(0,603)$ & $-0,052(0,597)$ & $-0,019(0,841)$ & $0,041(0,724)$ \\
\hline$<2000$ & $0,182(0,150)$ & $-0,125(0,328)$ & $-0,261(0,036)^{\star}$ & $-0,097(0,537)$ \\
\hline$>2000$ & $-0,043(0,783)$ & $-0,058(0,707)$ & $-0,039(0,803)$ & $0,033(0,852)$ \\
\hline HbsAg & $0,062(0,525)$ & $0,055(0,572)$ & $-0,007(0,943)$ & $-0,022(0,847)$ \\
\hline$<1000$ & $0,073(0,814)$ & $0,244(0,422)$ & $0,271(0,370)$ & $-0,151(0,747)$ \\
\hline$>1000$ & $0,024(0,820)$ & $0,014(0,893)$ & $-0,035(0,734)$ & $-0,061(0,614)$ \\
\hline HbeAg & $-0,104(0,285)$ & $-0,073(0,457)$ & $0,005(0,958)$ & $-0,080(0,491)$ \\
\hline Negative & $0,028(0,784)$ & $-0,083(0,428)$ & $-0,103(0,318)$ & $0,545(p<0,001)^{*}$ \\
\hline Positive & $-0,365(0,220)$ & $-0,449(0,124)$ & $-0,195(0,522)$ & $-0,187(0,583)$ \\
\hline
\end{tabular}

*. Correlation is significant at the 0.05 level; Kyn/Trp: kynurenine/tryptophan ratio. 
with HBV confirm that neopterin can be used as the marker of the presence of disease. Since neopterin levels in body fluids are reported to give an information about the current state of cellular immune response, neopterin is used for the prediction of the progression and outcome of the several pathological circumstances like viral infections, inflammatory diseases, various malignant disorders, neurological, and cardiovascular diseases [2022]. Neopterin can therefore be used as an early marker of inflammation for liver diseases such as acute and chronic hepatitis [23].

In a study investigating the role of neopterin in the early diagnosis of an infection, HBsAg positive patients had higher percentage of neopterin compared to control groups [5]. When we evaluated the HBsAg positive patients according to their qHBsAg levels $(<1000$ and $>1000)$, neopterin levels in both groups were significantly higher compared to control. However, there was no difference between the HBsAg groups, suggesting that neopterin may be insufficient to differentiate the quantitative $\mathrm{HbsAg}$ levels in patients.

In HBV infection, HBV DNA levels are used to identify patients with high viral replication levels who can, thus, benefit from an antiviral treatment and to monitor their treatment. In the study conducting by Kaleli et al., HBV DNA $>5 \mathrm{pg} / \mathrm{mL}$ and HBeAg positive patients classified as replicative group and neopterin levels were reported to be higher compared to a control group in carriers of $\mathrm{HBeAg}$ positive HBV. However, since there was no correlation found between neopterin and HBV DNA levels in these patients, it was reported that neopterin levels might not be correlated with the amount of viral load but with the presence of viral replication [24]. In our study, we compared neopterin levels according to HBV DNA load ( $<2000$ and $>2000$ ) but did not find any difference between the groups. Otherwise, our findings of significant increase in ALT and AST levels in patients with high viral load were similar to previous studies [25]. Neopterin levels were not correlated with ALT in chronic hepatitis patients similar to other studies [26, 27].

According to Kalkan et al., the identification of high neopterin concentrations in $\mathrm{HbeAg}$ positive chronic hepatitis B patients suggests a correlation between HbeAg positivity and neopterin levels [26]. In our study, serum neopterin levels were found to be significantly higher in both patient groups with HBeAg positive and negative compared to control. No correlation was found between HbeAg positivity and neopterin levels; on the contrary, a significant positive correlation was detected with $\mathrm{HbeAg}$ negativity $(\mathrm{r}=0.545, \mathrm{p}<0.001)$.
Serum neopterin levels were reported to be relatively high in patients with liver cirrhosis independently of their etiologies compared to non-cirrhosis patient and control groups [28]. Results of a pediatric study shows that serum neopterin levels of patients with cirrhosis were significantly higher than patients with chronic hepatitis. Positive correlation has been found between serum neopterin levels and liver HAI scores in chronic hepatitis and cirrhosis patients. Macrophages and lymphocytic cell infiltration in fibrous bands have been shown in the liver of patients with cirrhosis [29]. Exposure of liver tissue macrophages to high level endotoxins leads to overproduction of inflammatory mediators and eventually leads to liver damage. Neopterin released from active macrophages by stimulation with endotoxins may explain high neopterin levels in chronic liver diseases [29, 30]. In present study, we found a significant positive correlation between neopterin and HAI level in patients $(r=0.391$, $\mathrm{p}=0.018$ ). Moreover, when compared to patients with HAI $<4$, we also found that serum neopterin concentration was markedly increased in patients with $\mathrm{HAI} \geq 4$. Therefore, in the cirrhotic patients, it is thought that neopterin levels can be used as an indicator of the presence and severity of inflammation and necrosis of the liver. Hence, it is thought that neopterin can provide complementary information to the biopsy and improve clinical assessment.

In our study, IDO was evaluated as another marker induced by IFN-y, and IDO mediated Trp degradation in patients with HBV was found to be significantly higher compared to the control. Moreover, a significant positive correlation was detected between serum neopterin levels and IDO activity in the patient group ( $\mathrm{r}=0.304, \mathrm{p}=0.011$ ). This correlation between the neopterin concentration and IDO activity indicates an increased endogenous IFN-y increase. These findings suggest that Trp degradation in HBV patients is caused by activation of IDO rather than TDO. Under normal physiological conditions, approximately $90 \%$ of Trp degradation to Kyn occurs in liver via TDO and $<5-10 \%$ occurs extrahepatically via IDO [11]. However, the extrahepatic Kyn pathway becomes quantitatively significant in immune activation. Since TDO activity is inhibited in liver diseases, IDO-induced Trp depletion can be prevented to some extent and gradually increase with the severity of liver dysfunction [10]. In our patient group, Trp levels were slightly higher than the control group. While normally the level of Trp is expected to increase significantly, unchanged Trp levels can thus be explained by the activity of IDO rather than TDO.

IDO plays an important role in stimulation of immune tolerance during infection, pregnancy, transplantation, autoimmunity, and cancer. IDO, stimulated during 
inflammation by IFN-y and/or other inflammatory cytokines, induces regulatory $\mathrm{T}$ cells and increases immune tolerance by inhibiting $\mathrm{T}$ cell activation and proliferation or causing Trp deprivation and/or accumulation of Trp catabolites [31]. Depletion of Trp via IDO is also a part of cytostatic and antiproliferative activity mediated by IFN- $y$ in cells [32]. IDO starves invaders by depleting Trp and contributes to immunosuppression with microorganisms that are not cleared during the acute infection [33]. Depletion of Trp via IDO activation inhibits protein biosynthesis and afterwards the growth of pathogens and proliferating cells. Therefore, increased Kyn/Trp conversion is accepted as defense mechanism during the immune response and limits the growth of intracellular pathogens, infected cells, or malign cells [34, 35].

In HBV patients, we have shown that the Kyn/Trp ratio and Kyn levels are high due to increased immune response. Moreover, a significant correlation between IDO activity and AST or ALT levels were found in patients with low HBV DNA load $(<2000)$ but not for the high replicated group. Mao et al. have shown that expression of an enzymatically inactive IDO mutant did not inhibit HBV replication, and Trp supplementation in culture completely restored HBV replication in IDO-expressing cells, and, thus, suggested that the antiviral effect elicited by IDO is mediated by Trp deprivation [36].

Trp is an essential amino acid that directly affects the regulation of albumin synthesis in the liver [37]. In our study, we found a significant negative correlation between albumin levels and IDO activity in patients with HBV $(\mathrm{r}=-0.394, \mathrm{p}<0.001)$ due to the increased metabolism of Trp to Kyn. Normally, other amino acids need to be supplied in sufficient amounts for albumin synthesis. However, in animal experiments, it was found that the administration of essential amino acids without Trp did not correct the synthesis of albumin [38]. Decreased albumin synthesis may also be due to hepatocyte disorder. However, the lack of albumin synthesis is not seen at the same rate in all patients with liver cirrhosis and abnormal liver function tests [39]. In the light of the information mentioned above, decreased albumin synthesis and increased Trp degradation via the Kyn pathway may be a result of immune activation as well as hepatocyte damage.

Although studies on IDO activity in hepatitis B patients are limited, there are many studies on patients with hepatitis $C$ evaluating IDO activity. In a study, Trp and Kyn values measured in hepatitis $C$ patients were relatively lower than hepatitis B patients and healthy subjects and the difference with healthy subjects was found to be significant [40]. Higashitani et al. have demonstrated that systemic IDO activity is enhanced in chronic hepatitis $C$ patients, and this activity is influenced by HAI [31]. Similarly, in our study, HBV patients with HAI $\geq 4$ on liver biopsy were found to have higher IDO activity. Therefore, IDO can also provide additional prognostic marker for monitoring hepatocyte damage to improve clinical assessment.

\section{Conclusion}

In the clinic, the increase in the concentration of neopterin is identified in different pathological circumstances related to the cellular immune response like viral infections, inflammatory diseases, neurological, and cardiovascular diseases. Although different results regarding the relationship between routinely used HBV markers in clinic and neopterin levels, it is a common opinion that neopterin levels can be used as markers of disease. In our study, significantly higher neopterin levels in HBV infected compared to healthy volunteers also indicating the role of neopterin in the diagnosis the disease. Similarly, a significant increase of serum neopterin levels in patients with HAI $\geq 4$ suggests that neopterin could be an important indicator for evaluation of the presence and severity of inflammation and necrosis of the liver in the cirrhotic patients.

IDO is thought to have a physiological antiviral function in HBV infection. Along with its immuneregulating and antiviral functions, IDO plays an immunomodulatory role in virus-host interaction. According to our results, Trp metabolism via IDO was associated with the disease activity and liver histological activity in HBV patients. Since the significant correlation found between albumin levels and IDO activity in HBV patients, decreased albumin synthesis was thought to result from increased Trp degradation via Kyn pathway. Moreover, because of the significant positive correlation found between neopterin levels and IDO activity in patient group, co-evaluation of both markers may provide better discrimination for hepatitis B disease stage.

In conclusion, based on the study result, serum neopterin levels and IDO activity are suggested to provide complementary immunological information for monitoring hepatocyte damage to identify the presence of hepatitis B and can be used as prognostic markers in HBV disease. 


\section{Abbreviations}

Alanine aminotransferase (ALT); Aspartate aminotransferase (AST); Gama-glutamil transpeptidaz (GGT); Hepatitis B e-antigen (HBeAg); Hepatitis B surface antigen (HBsAg); Hepatitis B virus (HBV); Hepatitis B e-antibodies (AntiHBe); Histological activity index (HAI); Indoleamine 2,3-dioxygenase (IDO); Interferon gamma (INF-g); Kynurenine (Kyn); Trp 2,3-dioxygenase (TDO); Tryptophan (Trp).

Acknowledgments: Special thanks to F. Esra Önen Bayram and Cemre Şahin Şenyüz for their helpful advice on various technical issues.

Conflict of interest: Authors state no conflict of interest.

\section{References}

1. World Health Organization. Hepatitis B fact sheet. Geneva: WHO; 2017. Available from: http://www.who.int/mediacentre/ factsheets/fs204/en/. Cited 7 June 2018.

2. EASL. 2017. Clinical Practice Guidelines on the management of hepatitis B virus infection. Available at: https://easl.eu/ wp-content/uploads/2018/10/HepB-English-report.pdf

3. Gitlin N. Hepatitis B: Diagnosis, prevention, and treatment. Clin. Chem.1997; 43(43(8):1500-6.

4. Terrault NA, Lok AS, McMahon BJ, Chang KM, Hwang JP, Jonas $M M$, et al. Update on prevention, diagnosis, and treatment of chronic hepatitis B: AASLD 2018 hepatitis B guidance. Hepatology. 2018 Apr;67(4):1560-99.

5. Ünüvar S, Aslanhan H, Tanrıverdi Z, Karakuş F. The relationship between neopterin and hepatitis $B$ surface antigen positivity. Pteridines. 2018;29(1):1-5.

6. Hoffmann G, Wirleitner B, Fuchs D. Potential role of immune system activation-associated production of neopterin derivatives in humans. Inflamm Res. 2003 Aug;52(8):313-21.

7. Woloszczuk W, Troppmair J, Leiter E, Flener R, Schwarz $M$, Kovarik J, et al. Relationship of interferon-gamma and neopterin levels during stimulation with alloantigens in vivo and in vitro. Transplantation. 1986 Jun;41(6):716-9.

8. Sahin TT, Yuksel O, Girgin G, Sipahi H, Dikmen K, Azili C, et al. Is neopterin level a predictive and differential biomarker in patients with thyroid disorders? J Endocrinol Invest. 2009 Feb;32(2):147-9.

9. Baydar T, Yuksel O, Sahin TT, Dikmen K, Girgin G, Sipahi H, et al. Neopterin as a prognostic biomarker in intensive care unit patients. J Crit Care. 2009 Sep;24(3):318-21.

10. Badawy AA, Guillemin G. The Plasma [Kynurenine]/ [Tryptophan] Ratio and Indoleamine 2,3-Dioxygenase: time for Appraisal. Int J Tryptophan Res. 2019 Aug;12:1178646919868978.

11. Badawy AA. Kynurenine pathway of tryptophan metabolism: regulatory and functional aspects. Int J Tryptophan Res. 2017 Mar;10:1178646917691938.
12. Bedossa P, Poynard T; The METAVIR Cooperative Study Group. An algorithm for the grading of activity in chronic hepatitis $C$. Hepatology. 1996 Aug;24(2):289-93.

13. Sipahi H, Girgin G, Inanici F, Ariogul S, Sahin G, Baydar T. Tryptophan degradation and neopterin levels by aging. Pteridines. 2013;24(1):33-9.

14. Tang LS, Covert E, Wilson E, Kottilil S. Chronic Hepatitis B Infection: A Review. JAMA. 2018 May;319(17):1802-13.

15. Tao Y, Wu D, Zhou L, Chen E, Liu C, Tang X, et al. Present and Future Therapies for Chronic Hepatitis B. Adv Exp Med Biol. 2020;1179:137-86.

16. Akhan S, Aynıŏlu A, Çağatay A, Gönen I, Günal O, Kaynar T, et al.. Kronik hepatit $B$ virusu infeksiyonunun yönetimi: Türk Klinik Mikrobiyoloji ve İnfeksiyon Hastalıkları Derneği Viral Hepatit Çalışma Grubu Uzlaşı Raporu. Klimik Derg. 2014;27(Özel Sayı 1):S2-18.

17. Ohtaki H, Ito H, Ando K, Ishikawa T, Hoshi M, Ando T, et al. Kynurenine production mediated by indoleamine 2,3-dioxygenase aggravates liver injury in HBV-specific CTLinduced fulminant hepatitis. Biochim Biophys Acta. 2014 Sep;1842(9):1464-71.

18. Reibnegger G, Fuchs D, Hausen A, Werner ER, WernerFelmayer $\mathrm{G}$, Wachter $\mathrm{H}$. Neopterin and viral infections: diagnostic potential in virally induced liver disease. Biomed Pharmacother. 1989;43(4):287-93.

19. Fuchs D, Hausen A, Reibnegger G, Werner ER, Wachter H. Neopterin in clinical medicine. Lancet. 1988 Mar;1(8587):702.

20. Sipahi H, Girgin G, Palabiyik SS, Tutkun E, Yilmaz OH, Baydar T. Possible changes of New-Generation inflammation markers with occupational lead exposure. J Occup Health. 2017 Jul:59(4):345-51.

21. Cinici E, Palabiyik SS, Sipahi H, Baydar T. Nitrite, neopterin levels and tryptophan degradation in allergic conjunctivitis. Int Ophthalmol. 2018 Oct;38(5):1871-8.

22. Murr C, Widner B, Wirleitner B, Fuchs D. Neopterin as a marker for immune system activation. Curr Drug Metab. 2002 Apr;3(2):175-87.

23. Samsonov MI, Golban TD, Nasonov EL, Masenko VP. [Serum neopterin in hepatitis B]. Klin Med (Mosk). 1992 Mar-Apr;70(34):40-2.

24. Kaleli I, Demir M, Cevahir N, Yilmaz M, Demir S. Serum neopterin levels in patients with replicative and nonreplicative HBV carriers. BMC Infect Dis. 2006 Oct;6(1):157.

25. Kwon H, Lok AS. Hepatitis B therapy. Nat Rev Gastroenterol Hepatol. 2011 May;8(5):275-84.

26. Kalkan A, Ozden M, Akbulut H. Serum neopterin levels in patients with chronic hepatitis B. Jpn J Infect Dis. 2005 Apr;58(2):107-9.

27. Daito K, Suou T, Kawasaki H. Clinical significance of serum and urinary neopterin levels in patients with various liver diseases. Am J Gastroenterol. 1992 Apr;87(4):471-6.

28. Wilmer A, Nölchen B, Tilg H, Herold M, Pechlaner C, Judmaier G, et al. Serum neopterin concentrations in chronic liver disease. Gut. 1995 Jul;37(1):108国12.

29. Gulcan EM, Tirit I, Anil A, Adal E, Ozbay G. Serum neopterin levels in children with hepatitis-B-related chronic liver disease and its relationship to disease severity. World J Gastroenterol. 2008 Nov;14(44):6840-3.

30. Kmieć Z. Cooperation of liver cells in health and disease. Adv Anat Embryol Cell Biol. 2001;161:III-XIII. 
31. Higashitani K, Kanto T, Kuroda S, Yoshio S, Matsubara T, Kakita N, et al. Association of enhanced activity of indoleamine 2,3-dioxygenase in dendritic cells with the induction of regulatory $\mathrm{T}$ cells in chronic hepatitis $\mathrm{C}$ infection. J Gastroenterol. 2013 May;48(5):660-70.

32. Schröcksnadel K, Wirleitner B, Winkler C, Fuchs D. Monitoring tryptophan metabolism in chronic immune activation. Clin Chim Acta. 2006 Feb;364(1-2):82国90.

33. Mehraj V, Routy JP. Tryptophan Catabolism in Chronic Viral Infections: Handling Uninvited Guests. Int J Tryptophan Res. 2015 Aug;8:41-8.

34. Ozaki Y, Edelstein MP, Duch DS. Induction of indoleamine 2,3-dioxygenase: a mechanism of the antitumor activity of interferon gamma. Proc Natl Acad Sci USA. 1988 Feb;85(4):1242-6.

35. Pfefferkorn ER. Interferon gamma blocks the growth of Toxoplasma gondii in human fibroblasts by inducing the host cells to degrade tryptophan. Proc Natl Acad Sci USA. 1984 Feb;81(3):908-12.

36. Mao R, Zhang J, Jiang D, Cai D, Levy JM, Cuconati A, et al. Indoleamine 2,3-dioxygenase mediates the antiviral effect of gamma interferon against hepatitis $B$ virus in human hepatocyte-derived cells. J Virol. 2011 Jan;85(2):1048-57.

37. Pennisi VR, Pennisi VM, Wyatt J, Capozzi A. The relationship of tryptophan and albumin in the acute burn patient: preliminary observations. Burns. 1978;5(2):174-80.

38. Yap SH, Hafkenscheid JC, van Tongeren JH. Important role of tryptophan on albumin synthesis in patients suffering from anorexia nervosa and hypoalbuminemia. Am J Clin Nutr. 1975 Dec;28(12):1356-63.

39. Rothschild MA, Oratz M, Zimmon D, Schreiber SS, Weiner I, Van Caneghem A. Albumin synthesis in cirrhotic subjects with ascites studied with carbonate-14C. J Clin Invest. 1969 Feb;48(2):344-50.

40. Cozzi A, Zignego AL, Carpendo R, Biagiotti T, Aldinucci A, Monti $M$, et al. Low serum tryptophan levels, reduced macrophage IDO activity and high frequency of psychopathology in HCV patients. J Viral Hepat. 2006 Jun;13(6):402-8. 